\title{
Dependence of the Depth-Dose Distributions and Ranges of Proton Beams in Water Medium on Geant4 Parameters
}

\author{
A. ToK*, M. Bektasoglu and H. Arslan \\ Sakarya University, Department of Physics, Sakarya, Turkey
}

\begin{abstract}
The aim of this work is to investigate the influence of the Geant4 parameters on the range and the depth-dose distribution (Bragg curve) of proton beams with incident energies of 90, 150 and $230 \mathrm{MeV}$ injected upon a water medium, using the Geant4 simulation package. The simulated Bragg peak ranges and the depth-dose profiles have been compared with the ones obtained from the National Institute of Standards and Technology and the experiments, respectively. It has been seen that the Geant4 parameters, such as the step size and production cuts, under study have non-negligible effects on the range and the depth-dose distribution of incident proton energies of interest, especially at 150 and $230 \mathrm{MeV}$.
\end{abstract}

DOI: 10.12693/APhysPolA.130.438

PACS/topics: 87.55.ne, 87.10.Rt

\section{Introduction}

In proton therapy, proton beams with energies ranging from $\sim 60$ to $250 \mathrm{MeV}$ are used for treatment of cancerous cells (see, for instance [1-2]). The major goal of this therapy is to maximize the radiation dose transferred to the tumorous cells, while minimizing the dose transferred to the healthy tissue surrounding the tumorous ones.

Photon beams used in radiotherapy have physical and biological limitations in comparison with the proton beams. When photon beams interact with a tissuelike matter, for instance water, the dose distribution increases within the first few centimetres of the medium until it reaches a maximum and then it decreases according to the exponential law. This behaviour indicates that the maximum dose is transferred to the near surface. Proton beams, on the other hand, show a much more favourable behaviour due to the reduced lateral scattering and longitudinal straggling. Furthermore, one of the fundamental behaviors of the proton beams in matter is their exhibition of a steep maximum dose with a narrow peak, known as the Bragg peak close to the end of their range [3]. The reason for the appearance of the Bragg peak is the fact that when a fast proton, like other fast charged hadron, goes through a medium, it loses energy since it ionizes the atoms of the matter, and the interaction cross-section increases as its energy decreases. This physical property of the proton beams makes them more appropriate to be used in the treatment of tumors due to the fact that the healthy tissues surrounding the tumorous region is less affected by the radiation damage.

In the present work, we have used the Geant4 (for GEoemetry And Tracking) simulation package [4] to study the effects of two Geant4 parameters, namely the step size and production cut, on the depth-dose profiles and ranges of the proton beams with initial energies of 90, 150 and $230 \mathrm{MeV}$, incident upon a water phantom.

\footnotetext{
* corresponding author; e-mail: demirfizas@gmail.com
}

The step size and production cuts correspond to the distance to next interaction and production threshold for secondary particles (gammas, electrons and positrons) after EM interactions [5], respectively. Results of the simulations for the depth-dose profiles and ranges have been compared with the experimental and National Institute of Standards and Technology (NIST) data, respectively.

\section{Simulations}

Monte Carlo simulations for interactions of the protons with the water phantom have been performed using Geant4, release 10.0. The phantom has been considered to be a cubic box with dimensions $40 \times 40 \times 40 \mathrm{~cm}^{3}$. The target representing the water phantom and the event schemes for several protons are illustrated in Fig. 1. The ionization potential of water has been set to $75 \mathrm{eV}$, in accordance with the ICRU reports [6]. Simulations have been rerun for different Geant4 parameters of range (production) and step cuts, which correspond to the production threshold for secondary particles and to the distance to the next interaction, respectively. For the range cuts (step sizes) four (three) values, which are 1, 0.1, 0.01 and $0.001 \mathrm{~mm}(1,0.1$ and $0.01 \mathrm{~mm})$, have been used. Monoenergetic 500000 protons with initial energies of 90 , 150 and $230 \mathrm{MeV}$ have been injected upon the phantom for each run of the simulations. The number of incident particles have been chosen such that they are both sufficiently large to reduce the fluctuations and, meanwhile, since the computational time increases with number of simulated particles, adequately small to keep the computational time low.

Geant4 provides a comprehensive set of physics processes to model the interaction of particles with matter. For electromagnetic interactions the standard package, which includes multiple scattering of protons interacting with the atomic nuclei, in addition to the straggling of protons with the atomic electrons, has been used. For hadronic interactions the elastic and binary_ion models have been utilized for elastic and inelastic scattering, respectively [7]. 


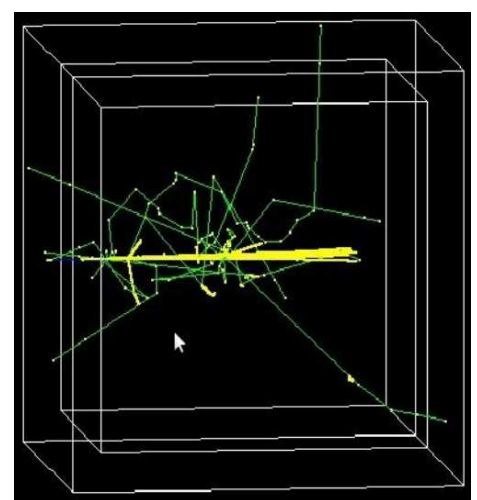

Fig. 1. The Geant4 water phantom and sample event schemes.

\section{Results and discussion}

Influence of the step cuts of interest on the proton range in water as a function of the range cut is shown in Fig. 2a, b and c for proton energies of 90,150 and $230 \mathrm{MeV}$, respectively. It can be concluded from Fig. 2a that, both the range and step cuts have no significant effect on the simulation results for the range of protons with low energies, namely at $90 \mathrm{MeV}$. For higher proton energies, the influence becomes more remarkable (see Fig. $2 \mathrm{~b}$ and c, for 150 and $230 \mathrm{MeV}$, respectively). In the case of relatively higher energies, simulation results for the range converge towards the NIST range $[8]$ as the range cut (and/or the step cut) decreases. It should be noted that, the increased accuracy at very low cuts costs a significant increase in the simulation time. In the present study it has been observed that setting both the range and step cut values as $0.01 \mathrm{~mm}$ seems to be ideal from the point of view of not only agreement with the NIST values but also a reasonable computational time.

Having determined the ideal cut value, the depth-dose profile of 90,150 and $230 \mathrm{MeV}$ protons have also been simulated using the 0.01 cut, both for the range and step cuts. Result of the simulated depth-dose profiles for the incident proton energies of interest are illustrated in Fig. 3, together with the experimental values [9]. It can be seen from the figure that the cut value of $0.01 \mathrm{~mm}$ is sufficient for obtaining a reasonable agreement between the simulated and experimental depth-dose curves of the protons. The number of bins used in the simulated distributions can be considered as the main reason for the discrepancy between the simulated and experimental data.

\section{Conclusions}

Effects of two user defined Geant4 parameters, the step size and the range cut, on the range and depth-dose distribution of protons with energies of 90,150 and $230 \mathrm{MeV}$ have been studied in water medium using the Geant 4 simulation package. For this purpose, first of all, the simulations have been performed to obtain the proton ranges for the range (step) cuts of 1, 0.1, 0.01 and $0.001 \mathrm{~mm}$ $(1,0.1$ and $0.01 \mathrm{~mm})$, for each proton energy of interest, separately. It has been seen that agreement between

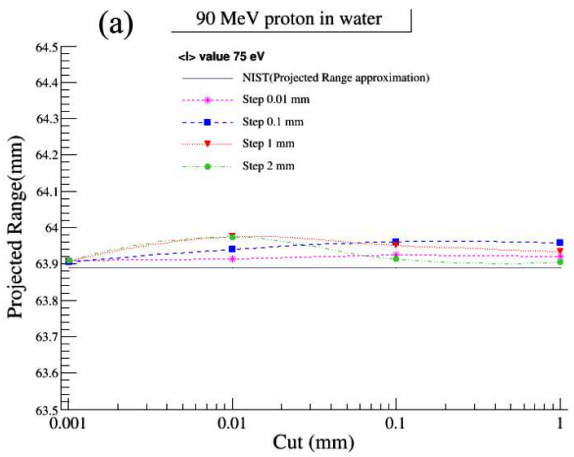

(b) $150 \mathrm{MeV}$ proton in water

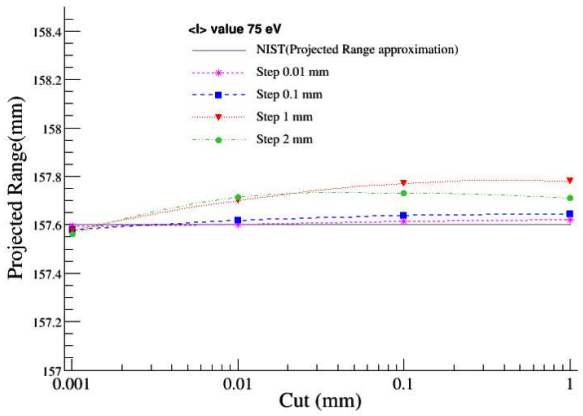

(c) $230 \mathrm{MeV}$ proton in water

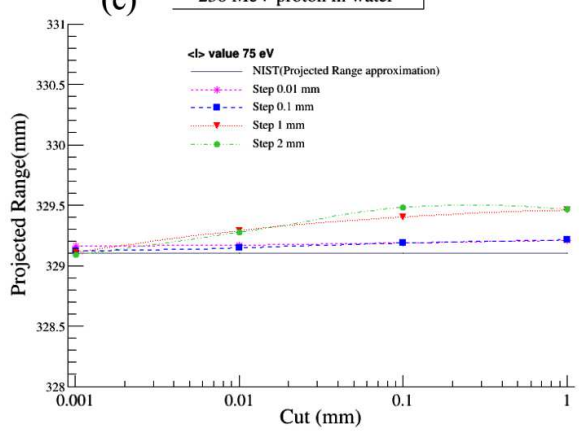

Fig. 2. Influence of various step cuts on the proton range in water as a function of the range cut for proton beam energies of (a) $90 \mathrm{MeV}$ (b) $150 \mathrm{MeV}$ and (c) $230 \mathrm{MeV}$.

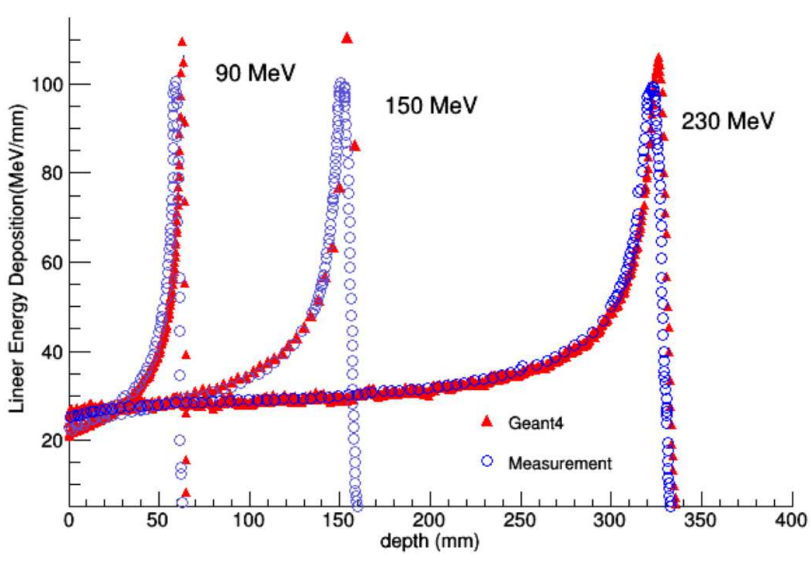

Fig. 3. The simulated (filled triangles) and experimental (open circles) depth-dose profiles of proton beams with various energies. 
the simulated and NIST ranges becomes more satisfactory as the range cut decreases for all the step cuts of interest with the realization that the finer step cuts also yield better agreement with the NIST ranges for a specific range cut. In addition, reducing the cut values results in a significant increase in simulation time. Therefore, the range and step cut of $0.01 \mathrm{~mm}$ has been concluded to be an optimal value both for obtaining an agreement with the NIST data and for a decreased simulation time. Furthermore, the simulated depth-dose profiles of 90, 150 and $230 \mathrm{MeV}$ protons using the above mentioned value as both the range and step cut have been seen to be in reasonable agreement with the experimental data.

\section{References}

[1] H. Paganetti, Technol. Cancer Res. Treat. 2, 413 (2003).

[2] C. Bert, R. Engelhart-Cabilic, M. Durante, Med. Phys. 39, 1716 (2012).

[3] W.H. Bragg, R. Kleeman, Philos. Mag. 8, 726 (1904).

[4] S. Agostinelli, J. Allison, K. Amako, J. Apostolakis, H. Araujo, P. Arce, M. Asai, D. Axen, S. Banerjee, G. Barrand, F. Behner, L. Bellagamba, J. Boudreau, L. Broglia, A. Brunengo, H. Burkhardt, S. Chauvie, J. Chuma, R. Chytracek, G. Cooperman, G. Cosmo, P. Degtyarenko, A. Dell'Acqua, G. Depaola, D. Dietrich, R. Enami, A. Feliciello, C. Ferguson, H. Fesefeld, G. Folger, F. Foppiano, A. Forti, S. Garelli, S. Giani, R. Giannitrapani, D. Gibin, J.J. Gomez Cadenas, I. Gonzalez, G. Gracia Abril, G. Greeniaus, W. Greiner, V. Grichine, A. Grossheim, S. Guatelli, P. Gumplinger, R. Hamatsu, K. Hashimoto, H. Hasui, A. Heikkinen, A. Howard, V. Ivanchenko, A. Johnson, F.W. Jones, J. Kallenbach, N. Kanaya, M. Kawabata, Y. Kawabata, M. Kawaguti, S. Kelner, P. Kent, A. Kimura, T. Kodama, R. Kokoulin, M. Kossov, H. Kurashige, E. Lamanna, T. Lampen, V. Lara, V. Lefebure, F. Lei, M. Liendl, W. Lockman, F. Longo, S. Magni, M. Maire, E. Medernach, K. Minamimoto, P. Mora de Freitas, Y. Morita, K. Murakami, M. Nagamatu, R. Nartallo, P. Nieminen, T. Nishimura, K. Ohtsubo, M. Okamura, S. O'Neale, Y. Oohata, K. Paech, J. Perl, A. Pfeiffer, M.G. Pia, F. Ranjard, A. Rybin, S. Sadilov, E. Di Salvo, G. Santin, T. Sasaki, N. Savvas, Y. Sawada, S. Scherer, S. Sei, V. Sirotenko, D. Smith, N. Starkov, H. Stoecker, J. Sulkimo, M. Takahata, S. Tanaka, E. Tcherniaev, E. Safai Tehrani, M. Tropeano, P. Truscott, H. Uno, L. Urban, P. Urban, M. Verderi, A. Walkden, W. Wander, H. Weber, J.P. Wellisch, T. Wenaus, D.C. Williams, D. Wright, T. Yamada, H. Yoshida, D. Zschiesche, Nucl. Instrum. Methods Phys. Res. A 506, 250 (2003).
[5] L. Grevillot, T. Frisona, N. Zahra, D. Bertrand, F. Stichelbaut, N. Freud, D. Sarrut, Nucl. Instrum. Methods Phys. Res. B 268, 3295 (2010).

[6] ICRU Report, 49 (1993).

[7] Available at http://geant4.cern.ch/ collaboration/working_groups/electromagnetic/ physlist.shtml, 2015.

[8] Available at http://physics.nist.gov, 2015.

[9] Available at www.rptc.de/fileadmin/user_upload/ rptc/Jahresberichte/JB4_ENG_110713_v6.pdf, 2015. 